\title{
BMJ Open Ethnicity matters in perceived impacts and information sources of COVID-19 among mothers with young children in Australia: a cross-sectional study
}

\author{
Li Ming Wen (1) , 1,2,3,4,5 Huilan Xu, ${ }^{2}$ Danielle Jawad, ${ }^{2}$ Limin Buchanan, ${ }^{1,2,3,5}$ \\ Chris Rissel, ${ }^{1}$ Philayrath Phongsavan, ${ }^{1,4}$ Louise A Baur, ${ }^{1,4,5,6}$ Sarah Taki ${ }^{1,2,3,5}$
}

To cite: Wen LM, Xu H, Jawad D, et al. Ethnicity matters in perceived impacts and information sources of COVID-19 among mothers with young children in Australia: a cross-sectional study. BMJ Open 2021;11:e050557. doi:10.1136/ bmjopen-2021-050557

\section{- Prepublication history and} additional supplemental material for this paper are available online. To view these files, please visit the journal online (http://dx.doi.org/10.1136/ bmjopen-2021-050557)

Received 23 February 2021 Accepted 02 November 2021

Check for updates

(C) Author(s) (or their employer(s)) 2021. Re-use permitted under CC BY-NC. No commercial re-use. See rights and permissions. Published by BMJ.

For numbered affiliations see end of article.

Correspondence to

Professor Li Ming Wen;

Liming.Wen@health.nsw.gov.au

\section{ABSTRACT}

Objectives This study aimed to investigate perceived impacts, ways of communication with professionals and information sources related to COVID-19, and explore whether these impacts or information sources were associated with ethnicity that is, language spoken at home.

Design A cross-sectional study.

Setting Sydney, Australia during the period from March to October 2020.

Participants Mothers of young children participating in an existing trial.

Outcome measures Mothers were asked to respond to a set of survey questions related to COVID-19 via telephone. The questions included a mental health scale, and how they communicated with health professionals and their information sources related to COVID-19 during the COVID-19 pandemic.

Results 0 537 mothers who completed the survey (81\% response rate), $45 \%$ reported they spoke a language other than English at home. Overall, 136 (26\%) reported experiencing mental distress. 234 (44\%) reported that COVID-19 affected the way they receive and communicate health-related information with health professionals, especially for those from non-English speaking backgrounds with an adjusted odds ratio (AOR) $1.58(95 \% \mathrm{Cl} 1.10$ to 2.27$)$. They were less likely to use a face-to-face service (AOR $0.55,95 \% \mathrm{Cl} 0.37$ to 0.80 ) and more likely to use social media (AOR $2.11,95 \% \mathrm{Cl} 1.40$ to 3.17) for health-related information. Regarding sources of COVID-19-related information, mothers from non-Englishspeaking backgrounds were more likely to rely on family members (AOR 1.49, 95\% $\mathrm{Cl} 1.01$ to 2.19) and social media (AOR 3.34, 95\% Cl 2.05 to 5.43).

Conclusions COVID-19 has significantly impacted mothers with young children in regard to their mental health, means of communication with health professionals and sources of health information. Mothers from nonEnglish-speaking communities were less likely to use a face-to-face service, and more likely to seek information from family members and social media. Appropriate health support for non-English-speaking community needs to take these factors into account.

Trial registration number ANZCTR:12618001571268. they
Strengths and limitations of this study

- This is the first study to report on the role of ethnicity in perceived impacts and information sources of COVID-19 among mothers with young children in Australia.

- The study highlights the importance of taking ethnicity into account in providing appropriate health support for mothers with young child from various backgrounds during the COVID-19 pandemic.

- The study could be limited by potential sample selection bias as a result of survey participants from an existing trial.

- Further qualitative research is required to understand why there were differences in sources used for information on COVID-19 and healthy lifestyle behaviours.

\section{INTRODUCTION}

The COVID-19 pandemic has had profound effects on communities globally. ${ }^{1}$ Since February 2021, this SARS-CoV-2 has engulfed the world with approximately 105 million confirmed cases and 2.2 million deaths. ${ }^{1}$ In Australia, by January 2021, over 28000 confirmed cases and 908 deaths have been reported. ${ }^{2}$ As a result, containment measures have included closure of or limited access to government and private offices, schools, shops, parks and non-essential workplaces. ${ }^{3}$ This has directly and indirectly impacted people's daily activities, social events, food availability, dietary quality, sleep cycle, screen time, employment, access to recreational locations and financial security. ${ }^{4-8}$

Recent studies have identified multiple aspects of life that have been influenced by the pandemic. For instance, a study of Canadian families with young children found that COVID-19 restrictions adversely affected daily routines, with reduced physical activity and increased screen time as well as an overall 
increased consumption of food and snacks. ${ }^{9}$ Such changes, triggered by stress eating, working from home, online home schooling and limited access to outdoor play areas, are likely to lead to lower quality of life which in turn may lead to long lasting health problems. ${ }^{5}{ }^{10}$ In addition to the impact on health behaviours, COVID-19 restrictions also present unique stressors that have placed a burden on mental health. ${ }^{11-15}$ In examining the impacts of social restrictions and distancing measures, a recent metaanalysis of 19 studies with 93569 participants reported a higher prevalence of stress $(8.1 \%-81.9 \%)$, psychological distress (34.43\%-38\%), depression (14.6\%-48.3\%), anxiety $(6.33 \%-50.9 \%)$ and post-traumatic stress disorder $(7 \%-53.8 \%)$ during the pandemic when compared with previously estimated 1-year prevalence rates prior to the pandemic. ${ }^{11}$ That review highlighted that more adverse psychological symptoms were exhibited among women, people under 40 years and those with existing mental health illnesses, unemployment and students. ${ }^{11}$

Despite the rapid escalation and repercussions of the COVID-19 pandemic across all populations, it has disproportionally affected disadvantaged and culturally and linguistically diverse (CALD) communities. According to recent provisional analyses, COVID-19-related death is significantly higher among CALD communities than those of white ethnicity. ${ }^{16} 17$ This finding indicates that the influence of COVID-19 on CALD communities can potentially exacerbate health inequalities in already vulnerable populations. ${ }^{18}$ The higher death rates from COVID-19 among CALD populations may be partly due to factors such as lower education, difficulty finding up to date information from trustworthy sources, lack of accessibility of translated materials and language barriers to access health services. ${ }^{19-21}$

Further, within Australia, CALD communities have lower levels of health literacy. ${ }^{22}$ It is therefore possible that these previously mentioned factors may influence the quality of health information CALD communities receive about COVID-19 and their ability to respond appropriately. An Australian study also showed that people with inadequate health literacy and those who spoke a language other than English at home, struggled to find and understand information on COVID-19 from government sources compared with those with adequate health literacy and who spoke English at home. ${ }^{23}$ In contrast, a recent study highlighted government websites as one of the most used and trusted sources of COVID-19-related information among people of white ethnicity. ${ }^{24}$ Moreover, the trust and choice of selected sources of information are influenced by several demographic factors such as ethnicity, age, religion, education and political affiliation. ${ }^{24} 25$

To date, there has been limited research examining the role of ethnicity related to the impacts of COVID-19 and accessing health information. The aims of this study were to investigate perceived impacts, means of communication with professionals and information sources related to COVID-19 among mothers with young children; and further explore whether these impacts or information sources were associated with ethnicity.

\section{METHODS}

\section{Study design}

We conducted a cross-sectional survey of mothers with young children participating in an existing study ${ }^{26}$ in Sydney, Australia from March to October 2020. The mothers had participated in a longitudinal study since 2017 , and the study protocol was published prior to the commencement of this trial. ${ }^{26}$ The recruitment process and first year outcomes of the original trial have been reported elsewhere. ${ }^{27}{ }^{28}$ Briefly, the trial aimed to investigate effectiveness of an early childhood obesity prevention using telephone support or text messages. This current study was part of the 3-year follow-up survey.

\section{Patient and public involvement}

The survey participants were originally recruited to a longitudinal study ${ }^{27} 28$ from antenatal clinics in eight hospitals of four local health districts in Sydney. For this current study the development of the research questions and outcome measures was partially informed by the intervention nurses through their telephone support consultations with the study participants as part of the original trial. ${ }^{28}$ The study participants played no role in the design of this study, but their informed consent was sought. Not participating in this current study had no effect on their participation in the original study. The summary results of this study will be disseminated to all the participants through mailouts or the website. The study participants and project team members are acknowledged in the acknowledgements section.

\section{Inclusion and exclusion criteria}

For the original study, women were eligible to participate if they were aged 16 years and over, able to communicate in English, had a mobile phone and lived in the recruitment areas of a local hospital. Women were excluded from the study if they had a severe medical condition or known major fetal anomalies based on medical advice.

\section{Survey respondents and data collection}

A total of 662 mothers remained in the existing trial when their children reached 3 years. The survey was conducted by a marketing survey company using computer-assisted telephone interviewing.

\section{Perceived COVID-19 impacts}

We measured the impacts that COVID-19 has had on families from four aspects including (1) changes in eating, physical activity and screen time behaviours, (2) mental health, (3) participating in research and (4) whether the pandemic influenced their ways of receiving health-related information. The mothers were asked to respond to five statements on whether their family: (1) ate more snacks, fruit and vegetables, (2) had more screen time, (3) had more physical activity and (4) were 
more likely to follow mealtime or bedtime routines during the COVID-19 pandemic. We used a 5-level Likert scale, 'Strongly disagree', 'Disagree', 'Neither agree nor disagree', 'Agree' and 'Strongly agree'. The responses to each of the statements were further dichotomised into 'having more' if they 'Strongly agree' or 'Agree' to the statement, or 'no changes' (see online supplemental document about questionnaire and coding frame).

The Patient Health Questionnaire-4 (PHQ-4) was used to assess mothers' mental health. ${ }^{29}$ The mothers were asked how often they were bothered by the following over the last 2 weeks: (1) nervous and anxious, (2) worrying, (3) depressed or hopeless, (4) little interest or pleasure in doing things. Mothers responded to each of the questions on a 4-point scale, ' 0 ' for 'Not at all' to ' 3 ' for 'Nearly every day'. The PHQ-4 total score ranges from 0 to 12 , with categories of psychological distress being normal (0-2), mild (3-5), moderate (6-8) and severe (9-12). It was further dichotomised into 'psychological distress' (mild to severe) and 'none'. Mothers were also asked how often they worried about family members and close friends on a 4-point scale. Responses were dichotomised into 'worrying about family' (for several days to nearly every day), or 'no worry' (online supplemental document).

Assessing the impact COVID-19 had on their research participation, mothers were asked whether COVID-19 negatively affected their desire to participate in research. Mothers responded to the question on a 5-level Likert scale, 'Not at all', 'A little bit', 'Moderately', 'Quite a bit' and 'Extremely'. We also dichotomised the responses into 'Affecting research participation' (Moderately to Extremely) and 'No affect' (Not at all or A little bit). The mothers were asked whether the COVID-19 pandemic affected the way they receive and communicate healthrelated information with health professionals. They were also asked how likely they would use the following modes to receive and communicate health-related information with health professionals: face to face, telephone, short message service, videoconference, website, social media and booklets or pamphlets. Mothers responded to each of the modes on a 5-level Likert scale, 'Unlikely', 'Possibly', 'Likely', 'Almost certain' and 'Certain'. The responses were categorised into 'Yes' and 'No', with 'Yes' referring to 'Likely', 'Almost certain' or 'Certain'.

\section{Sources of health information for changing behaviours during COVID-19 pandemic}

We asked about sources of health information related to three areas specific to COVID-19: (1) COVID-19-related information, (2) changing food and beverage behaviour and (3) changing physical activity from government officials, health professionals, family members, social media or educators respectively. The question allowed multiple responses (see online supplemental document).

\section{Mothers' demographics}

Mothers' demographic information, including their language spoken at home, was collected at baseline using standard questions from the New South Wales (NSW) Adult Population Health Survey. ${ }^{30}$ All mothers' demographic and socioeconomic information were categorised into groups.

\section{Statistical analysis}

Statistical analyses were carried out using Stata V.13 (StataCorp). All p values are two sided and statistical significance was set at the $5 \%$ level. Descriptive analysis was conducted to describe mothers' baseline demographic characteristics for those who completed 3-year survey. Pearson's $\chi^{2}$ tests were conducted to examine the associations between mothers' demographic characteristics and COVID-19 impact and sources of health information during COVID-19 pandemic. Number and percentage were reported.

Multiple logistic regression models were built to investigate the associations between mothers' language spoken at home and COVID-19 impact and sources of health information during COVID-19 pandemic. To identify potential confounding factors, mothers' demographic variables that were significant in Pearson's $\chi^{2}$ tests with $\mathrm{p}<0.25$ were entered in the multiple logistic regression models. The least significant variables were progressively dropped until only those with $\mathrm{p}<0.05$ remained. Variables dropped from the model were then entered into the model individually to assess confounding. Since the survey respondents were involved in an intervention trial, their group allocation was also adjusted in the final model. Adjusted ORs (AORs) with 95\% CIs were then calculated as a measure of the association.

\section{RESULTS}

Of 662 mothers remaining in the follow-up study, 537 completed the COVID-19 survey questions with a response rate of $81 \%$. Table 1 shows demographic characteristics of the survey respondents with $45 \%$ of the mothers speaking a language other than English at home.

Table 2 shows that the impacts on mental health were observed across the survey respondents, with $26 \%$ reporting psychological distress (including $18 \%$ with mild symptoms of mental distress, $6 \%$ moderate symptoms and $2 \%$ severe mental distress) and $59 \%$ worrying about their family. There was no significant difference in psychological distress between mothers from English-speaking or non-English-speaking backgrounds. Substantial proportions of mothers reported having more snacks $(46 \%)$ and no increase in fruit and vegetable consumption (55\%), in particular among English-speaking mothers. Overall, $77 \%$ of the mothers reported having more screen time while $60 \%$ reported no increase in physical activity, with no significant differences found between Englishspeaking and non-English-speaking mothers. Significantly higher proportions of English-speaking mothers reported impacts of the COVID-19 restrictions on their mealtime $(71 \%)$ or bedtime $(76 \%)$ routines. 
Table 1 Demographics of the survey respondents in Sydney, Australia, March and October 2020

\begin{tabular}{|c|c|}
\hline & 3-year survey \\
\hline Mothers' demographics & $\begin{array}{l}\text { Completed } \\
\text { n (\%) }\end{array}$ \\
\hline \multicolumn{2}{|l|}{ Age (years) } \\
\hline$<30$ & $143(27)$ \\
\hline$\geq 30$ & $394(73)$ \\
\hline \multicolumn{2}{|l|}{ Country of birth } \\
\hline Australia & $211(39)$ \\
\hline Overseas & $326(61)$ \\
\hline \multicolumn{2}{|l|}{ Language spoken at home } \\
\hline English & $296(55)$ \\
\hline Other & $241(45)$ \\
\hline \multicolumn{2}{|l|}{ Annual household income } \\
\hline$<A U S \$ 80000$ & $316(64)$ \\
\hline$\geq$ AUS $\$ 80000$ & $175(45)$ \\
\hline \multicolumn{2}{|l|}{ Employment status } \\
\hline Employed & $365(68)$ \\
\hline Other & $172(32)$ \\
\hline \multicolumn{2}{|l|}{ Marital status } \\
\hline Married/de-facto partner & $508(95)$ \\
\hline Other & $28(5)$ \\
\hline \multicolumn{2}{|l|}{ Education level } \\
\hline University & $381(71)$ \\
\hline Up to HSC/TAFE & $155(29)$ \\
\hline \multicolumn{2}{|l|}{ First time mother } \\
\hline Yes & $303(56)$ \\
\hline No & $234(44)$ \\
\hline
\end{tabular}

HSC, High School Certificate; TAFE, Technical and Further Education.

Table 2 also shows $44 \%$ reported that COVID-19 affected the way they receive and communicate healthrelated information with health professionals, especially those who spoke a language other than English, with an AOR (AOR 1.58, 95\% CI 1.10 to 2.27). They were less likely to use a face-to-face service (AOR 0.55, 95\% CI 0.37 to 0.80 ) and more likely to use social media (AOR 2.11, $95 \%$ CI 1.40 to 3.17 ) for health-related information.

Table 3 shows the sources of COVID-19-related information and information for changes in food and beverage consumption and physical activity behaviours during the COVID-19 pandemic. For the COVID-19-related information almost all of mothers $(97 \%)$ relied on government officials, followed by health professionals $(77 \%)$, family members $(67 \%)$, educators $(52 \%)$ and social media $(26 \%)$. However, mothers who spoke a language other than English were more likely to rely on family members (AOR 1.49, 95\% CI 1.01 to 2.19) and social media (AOR $3.34,95 \%$ CI 2.05 to 5.43 ).
Less than half of the survey respondents (48\%) reported accessing sources of information for changing physical activity from government officials and 27\% reported accessing such information from health professionals (table 3). However, non-English-speaking mothers were more likely to rely on government officials (AOR 2.23, 95\% CI 1.49 to 3.32) or health professionals (AOR 3.58, 95\% CI 2.34 to 5.50) compared with English-speaking mothers. Regarding information for changing food and beverages only, about $20 \%$ reported accessing information from government officials or health professionals. Non-English-speaking mothers were more likely to rely on government officials (AOR 6.68, 95\% CI 3.56 to 12.55) or health professionals (AOR 5.26, 95\% CI 3.11 to 8.89), compared with English-speaking mothers.

\section{DISCUSSION}

This cross-sectional survey conducted with mothers during the pandemic found that one in four reported having psychological distress, with more than half of the respondents being worried about their family regardless of ethnicity. The COVID-19 pandemic resulted in more snack consumption and more screen time. It also affected the way mothers receive and communicate health-related information with health professionals, especially those who spoke a language other than English. Non-Englishspeaking mothers were less likely to use a face-to-face service and more likely to use family and social media for health-related information. The survey also found that the majority of mothers relied on government officials and health professionals for COVID-19-related information regardless of ethnicity.

\section{Impact of COVID-19 on mental health}

On average, general mental distress among mothers with young child was moderate. Most reported feeling normal $(74 \%)$, or having a mild (18\%) degree of mental distress. Similar patterns were found among English-speaking and non-English-speaking mothers. The mental health status of our survey respondents appeared to be better than that of respondents of a survey that examined mental health status of the general Australian population during the pandemic with responses from 5158 Australian adults. ${ }^{31}$ A possible explanation could be due to the difference in data collection period. That study collected data from 1 April 2020 to 4 April 2020 while lockdown restrictions were occurring in Australia. ${ }^{32}$ Our survey collected data from March till October 2020 which covered periods from lockdown to easing of restrictions in Australia. The varying results also could be explained by the different tools used for assessing mental health and the age range and gender of our study participants.

Our findings are complementary to a previous study conducted in Germany, which showed similar psychological effects of the pandemic using the same PHQ4 tool. Analysis of the survey data collected from (27 March-6 April) reported that the majority $(78.3 \%)$ of respondents 
Table 2 Perceived impacts of COVID on mothers and associations with mothers' language spoken at home

\begin{tabular}{|c|c|c|c|c|c|}
\hline \multirow[b]{2}{*}{ Covid-19 impacts } & \multirow[b]{2}{*}{$\begin{array}{l}\text { Total } \\
\text { n (\%) }\end{array}$} & \multicolumn{4}{|c|}{ Language spoken at home } \\
\hline & & $\begin{array}{l}\text { English } \\
\text { n (\%) }\end{array}$ & $\begin{array}{l}\text { Other } \\
\text { n (\%) }\end{array}$ & $P$ value & $\begin{array}{l}\text { Other vs English } \\
\text { AOR }(95 \% \mathrm{Cl})\end{array}$ \\
\hline \multicolumn{6}{|l|}{ Perceived behavioural changes } \\
\hline Having more snacks & $244(46)$ & $154(52)$ & $90(38)$ & 0.001 & 0.52 (0.36 to 0.75$)$ \\
\hline $\begin{array}{l}\text { No increase in fruit and } \\
\text { vegetable consumption }\end{array}$ & $293(55)$ & $200(68)$ & $93(39)$ & $<0.0001$ & 0.31 (0.21 to 0.45$)$ \\
\hline Having more screen time & $412(77)$ & $235(80)$ & $177(74)$ & 0.107 & 0.71 (0.46 to 1.08$)$ \\
\hline No increase in physical activity & $319(60)$ & $174(59)$ & $145(61)$ & 0.728 & 1.07 (0.75 to 1.55$)$ \\
\hline Did not follow mealtime routine & $304(57)$ & $210(71)$ & $94(39)$ & $<0.0001$ & 0.27 (0.18 to 0.39$)$ \\
\hline Did not follow bedtime routine & $347(65)$ & $224(76)$ & $123(51)$ & $<0.0001$ & 0.34 (0.23 to 0.49$)$ \\
\hline \multicolumn{6}{|l|}{ Mental health } \\
\hline Psychological distress & $136(26)$ & $72(24)$ & $64(27)$ & 0.547 & 1.03 (0.68 to 1.55$)$ \\
\hline Worry about their family & $321(59)$ & $164(56)$ & $148(62)$ & 0.152 & 1.27 (0.88 to 1.82$)$ \\
\hline Participating research & $91(18)$ & $26(9)$ & $65(28)$ & $<0.0001$ & 2.20 (1.25 to 3.88$)^{x}$ \\
\hline The way of receiving health info & $234(44)$ & $115(39)$ & $119(50)$ & 0.014 & 1.58 (1.10 to 2.27$)$ \\
\hline \multicolumn{6}{|c|}{ The way of communicating with health professionals (multiple responses allowed) } \\
\hline Face to face & $344(65)$ & $207(71)$ & $137(57)$ & 0.001 & 0.55 (0.37 to 0.80$)$ \\
\hline Telephone & $406(76)$ & $237(81)$ & $169(71)$ & 0.006 & $0.70(0.44 \text { to } 1.11)^{x}$ \\
\hline SMS & $274(52)$ & $152(52)$ & $122(51)$ & 0.849 & 0.94 (0.66 to 1.35$)$ \\
\hline Video conference & $313(59)$ & $200(68)$ & $113(47)$ & $<0.0001$ & 0.45 (0.31 to 0.66$)$ \\
\hline Website & $277(52)$ & $158(54)$ & $119(50)$ & 0.342 & 0.79 (0.55 to 1.13$)$ \\
\hline Social media & $146(27)$ & $58(20)$ & $88(37)$ & $<0.0001$ & 2.11 (1.40 to 3.17 ) \\
\hline Booklets & $288(43)$ & $131(45)$ & $97(41)$ & 0.322 & 0.82 (0.57 to 1.19 ) \\
\hline
\end{tabular}

All models were adjusted for intervention allocations and employment status.

${ }^{*}$ Adjusted for intervention allocations, employment status and annual household income.

AOR, adjusted OR; SMS, short message service.

were concerned of the health consequences for their relatives. The average depressive and anxiety PHQ4 score was mild among participants, similar to our study. Additionally, the study highlighted that women had a significantly higher PHQ-4 score than men, highlighting the susceptibility of women to mental illness. ${ }^{33}$

Despite the low prevalence of mothers reporting mental distress in our current study, it is intuitive that COVID-19 pandemic restrictions would increase mental distress as evidenced by previous literature on the negative influences of public health crises on mental health. ${ }^{11-15}$ More than half of our study participants reported worrying about their family members. While government efforts to manage and eradicate COVID-19 continue, our society will also need to continue maintaining physical distancing, some social isolation and dealing with disruptions to life. Thus, it is imperative to intervene to protect communities, in particular those in high-risk groups such as women with young children, ${ }^{31}$ and other vulnerable communities such as non-Englishspeaking people, where a greater effort to communicate well is needed. Specifically, research needs to focus on better understanding, from a cultural lens, the cultural differences to help CALD communities deal with the changing contexts related to COVID-19, and how they see the role of governments and health professionals to help CALD communities interact with health systems (health literacy) ${ }^{23}$ Addressing this will increase the effectiveness of the COVID-19 response such as testing, following physical isolation restrictions and the uptake of vaccinations to effectively and successful manage subsequent waves of the pandemic.

\section{Sources used for health information}

Our study findings echo some previous studies in which it was also found that vulnerable populations are less likely to use face-to-face services and more likely to turn to other sources for health information such as religious leaders or family members and community leaders who may be important in many cultures. ${ }^{22}{ }^{24}$ Social media is an additional platform that has been used substantially by many people including CALD communities for healthrelated information. ${ }^{21}$ Using social media from nonmedical or non-government sources may be of concern, given the proliferation of readily available misinformation obtained via unreliable and unverified online social 
Table 3 Sources of COIVD-19-related information and health information for changing food and beverage and physical activity behaviours during the Covid-19 pandemic and their associations with mothers' language spoken at home

\begin{tabular}{|c|c|c|c|c|c|}
\hline & \multirow[b]{2}{*}{$\begin{array}{l}\text { Total } \\
\text { n (\%) }\end{array}$} & \multicolumn{4}{|c|}{ Language spoken at home } \\
\hline & & $\begin{array}{l}\text { English } \\
\text { n (\%) }\end{array}$ & $\begin{array}{l}\text { Other } \\
\text { n (\%) }\end{array}$ & $P$ value & $\begin{array}{l}\text { Other vs English } \\
\text { AOR }(95 \% \mathrm{Cl})\end{array}$ \\
\hline \multicolumn{6}{|c|}{ Sources of COVID-19-related information } \\
\hline Government officials & $517(97)$ & $288(98)$ & 229 (96) & 0.149 & $0.74(0.25$ to 2.21$)$ \\
\hline Health professionals & $410(77)$ & $229(78)$ & $181(76)$ & 0.556 & $0.87(0.57$ to 1.33$)$ \\
\hline Family members & $355(67)$ & $182(62)$ & $173(72)$ & 0.011 & 1.49 (1.01 to 2.19$)$ \\
\hline Social media & $136(26)$ & $35(12)$ & $101(42)$ & $<0.0001$ & $3.34(2.05 \text { to } 5.43)^{*}$ \\
\hline Educators & $278(52)$ & $141(48)$ & $137(57)$ & 0.031 & $1.31(0.91$ to 1.88$)$ \\
\hline \multicolumn{6}{|c|}{ Sources of health information for changing food and beverage behaviour } \\
\hline Government officials & $95(18)$ & $15(5)$ & $80(34)$ & $<0.0001$ & $6.68(3.56 \text { to } 12.55)^{\star}$ \\
\hline Health professionals & 99 (19) & $23(8)$ & 76 (32) & $<0.0001$ & 5.26 (3.11 to 8.89$)$ \\
\hline Family members & $102(19)$ & $18(6)$ & $84(35)$ & $<0.0001$ & 8.19 (4.65 to 14.43$)$ \\
\hline Social media & $47(9)$ & $6(2)$ & $41(17)$ & $<0.0001$ & $5.46(2.13 \text { to } 14.04)^{\star}$ \\
\hline Educators & $71(13)$ & $14(5)$ & $57(24)$ & $<0.0001$ & $4.01(2.05 \text { to } 7.86)^{*}$ \\
\hline \multicolumn{6}{|c|}{ Sources of health information for changing physical activity } \\
\hline Government officials & $258(48)$ & $114(39)$ & $144(60)$ & $<0.0001$ & $2.23(1.49 \text { to } 3.32)^{*}$ \\
\hline Health professionals & $145(27)$ & $48(16)$ & $97(41)$ & $<0.0001$ & 3.58 (2.34 to 5.50$) \dagger$ \\
\hline Family members & $134(25)$ & $45(15)$ & $89(37)$ & $<0.0001$ & 3.13 (2.03 to 4.81$)$ \\
\hline Social media & $60(11)$ & $12(4)$ & $48(20)$ & $<0.0001$ & 5.73 (2.91 to 11.31$)$ \\
\hline Educators & $96(18)$ & $30(10)$ & $66(28)$ & $<0.0001$ & 3.15 (1.93 to 5.16$)$ \\
\hline
\end{tabular}

All models were adjusted for intervention allocations and employment status.

*Adjusted for intervention allocations, employment status and annual household income.

†Adjusted for interventions allocations, employment status and marital status.

AOR, adjusted OR.

applications. ${ }^{3435}$ Recent evidence showed that two-thirds $(66 \%)$ of individuals encountered misinformation about COVID-19 on social media, ${ }^{36}$ an additional one-third $(36 \%)$ mentioned obtaining false information from news media coverage ${ }^{36}$ For instance, in the USA, misleading claims of a national lockdown fuelled panic buying of paper products and groceries resulting in food insecurity among vulnerable populations giving rise to mass hysteria and panic. ${ }^{37}$ While social media is highly used by populations of various backgrounds and can be a beneficial platform to share information, there is a need to improve social media literacy skills. More research needs to be conducted to identify relevant approaches to support CALD communities and improve navigating through credible information on digital or social media platforms.

Adverse psychosomatic outcomes are expected to increase especially among CALD communities due to social isolation, access barriers to health services, discrimination and racism, limited support networks, low English proficiency and poor digital literacy. ${ }^{38}$ These circumstances can potentially exacerbate existing health inequities for these vulnerable groups, thus perpetuating suboptimal health-seeking behaviours and poor engagement with healthcare professionals and the broader health system. As identified in our study, there is a cultural tendency to seek information from families, religious leaders and media, which highlights the importance of mitigating harm from digital 'infodemics' and tailoring messages to community values. ${ }^{38}$ There is a clear case for government agencies to take a leadership role in communicating with CALD communities using culturally appropriate methods, in particular to increase the uptake of COVID-19 vaccinations. In addition, a cross-sectional survey of Pennsylvania adults found that COVID-19 knowledge correlates with using trusted news sources (ie, government health websites). The study called for the use of government health websites, as well as monitoring and correcting misinformation presented by other sources (eg, Facebook) in order to maximise information dissemination and compliance with COVID-19-related public health recommendations. ${ }^{39}$

This study found that during the pandemic, the majority of people obtained COVID-19-related information from government officials and health professionals, but a much smaller proportion of mothers obtained health information for changing health behaviours from these sources. This does not necessarily mean that mothers did not receive diet and physical activity information or advice from government officials or health professionals. Rather, it may indicate that changing lifestyle 
behaviours was considered to be a low priority during the pandemic compared with navigating the various responses to COVID-19. Another reason might be that the majority of the population in Australia are less likely to have ever lived through a pandemic, and therefore, there is a sense of unfamiliarity in how to respond and react. Thus, the government played a major role in the response including constant changes in social restriction laws requiring the population to follow information from government officials.

Despite this, good nutrition and regular physical activity contribute to improving immunity. As such, WHO released guidelines on diet during the COVID-19 pandemic stating that 'good nutrition is crucial for health, particularly in times when the immune system might need to fight back'. ${ }^{40}$ However, there are currently very few culturally-appropriate programmes and resources that promote healthy eating and physical activity targeted at children aged under 5 years. ${ }^{41}$ Government and various health agencies will need to continue developing culturally appropriate resources and health-related behavioural change support material to mitigate the likelihood of long-term impacts of COVID-19 on chronic disease status.

\section{Strength and limitations}

Our survey is timely and specific to the impacts of COVID-19 during the pandemic. Mental health outcomes were measured with the PHQ-4 which is a psychometrically valid instrument and has been demonstrated as a valid screening tool in general populations. ${ }^{29}$ With regard to the limitations, our cross-sectional survey design hindered causal inference. The survey questions for perceived behaviour change, and information sources as impacted by COVID-19 were newly developed for the current study given that no validated instruments were available at the time we initiated this study. Additionally, our collection period ranged from March to October 2020 and within this period, Australia experienced a range of COVID-19 restrictions, from full lockdown to eased restrictions, to having varying levels of restrictions in place and in different states. ${ }^{32}$ We acknowledge that respondents' behavioural data only represent their state on the date they completed the survey with limited generalisability. Further qualitative research is required to understand why there were differences in sources used for information on COVID-19 and healthy lifestyle behaviours.

\section{CONCLUSION}

The response to COVID-19 has impacted on mothers with young children in regard to their mental health, means of communicating with health professionals and sources of health information. Mothers from CALD communities were less likely to use a face-to-face service, and more likely to seek information from family members and social media. Appropriate health support for CALD community needs to take these factors into account.
Author affiliations

${ }^{1}$ Sydney School of Public Health, The University of Sydney, Sydney, New South Wales, Australia

${ }^{2}$ Population Health Research \& Evaluation Hub, Sydney Local Health District, Camperdown, New South Wales, Australia

${ }^{3}$ Sydney Institute for Women, Children and Their Families, Sydney Local Health District, Sydney, New South Wales, Australia

${ }^{4}$ Charles Perkins Centre, The University of Sydney, Sydney, New South Wales, Australia

${ }^{5} \mathrm{NHMRC}$ Centre of Research Excellence in the Early Prevention of Obesity in Childhood (EPOCH), The University of Sydney, Sydney, New South Wales, Australia ${ }^{6}$ The Children's Hospital at Westmead Clinical School, The University of Sydney, Sydney, New South Wales, Australia

Acknowledgements We sincerely thank all the participating families in this study. We thank the members of the Steering Committee, Management Committee and working group for their advice and support. We wish to thank the project partners from the Sydney, South Eastern Sydney, South Western Sydney and Southern NSW Local Health Districts, in NSW, Australia.

Contributors LMW, CR and LAB conceived of the study. LMW prepared the first draft of the manuscript. $\mathrm{HX}$ conducted statistical analyses. DJ and LB contributed to literature review. CR, PP, LAB and ST contributed to revising the manuscript critically. All authors contributed to finalising the manuscript and approved the manuscript. LMW and HX are the guarantors for this study.

Funding This trial was funded by the NSW Health Translational Research Grant Scheme 2016 (ID number: TRGS 200) and the Australian National Health and Medical Research Council Partnership Project APP1169823.

Disclaimer We declare that the funder (NSW Health and NHMRC) played no role in the design and conduct of the study; collection, management, analysis, and interpretation of the data; preparation, review, or approval of the manuscript; and decision to submit the manuscript for publication.

Competing interests None declared.

Patient consent for publication Not applicable.

Ethics approval The trial was granted ethics approval by the Ethics Review Committee of Sydney Local Health District (Protocol No. X16-0360 and LNR/16/ RPAH/495 and Protocol No X18-0387 and HREC/18/RPAH/545). Written informed consent was obtained from all study participants.

Provenance and peer review Not commissioned; externally peer reviewed.

Data availability statement Data are available on reasonable request. Deidentified data and material can be available on request pending on ethics approval from Dec 302021 to Dec 302026.

Supplemental material This content has been supplied by the author(s). It has not been vetted by BMJ Publishing Group Limited (BMJ) and may not have been peer-reviewed. Any opinions or recommendations discussed are solely those of the author(s) and are not endorsed by BMJ. BMJ disclaims all liability and responsibility arising from any reliance placed on the content. Where the content includes any translated material, BMJ does not warrant the accuracy and reliability of the translations (including but not limited to local regulations, clinical guidelines, terminology, drug names and drug dosages), and is not responsible for any error and/or omissions arising from translation and adaptation or otherwise.

Open access This is an open access article distributed in accordance with the Creative Commons Attribution Non Commercial (CC BY-NC 4.0) license, which permits others to distribute, remix, adapt, build upon this work non-commercially, and license their derivative works on different terms, provided the original work is properly cited, appropriate credit is given, any changes made indicated, and the use is non-commercial. See: http://creativecommons.org/licenses/by-nc/4.0/.

ORCID iD

Li Ming Wen http://orcid.org/0000-0003-1381-4022

\section{REFERENCES}

1 Worldometer. Countries where COVID-19 has spread, 2020. Available: https://www.worldometers.info/coronavirus/countrieswhere-coronavirus-has-spread/ [Accessed 10 Dec 2020].

2 Coronavirus (COVID-19) current situation and case numbers. Australian government department of health. Australian government 
department of health, 2020. Available: https://www.health.gov. $\mathrm{au} /$ news/health-alerts/novel-coronavirus-2019-ncov-healthalert/coronavirus-covid-19-current-situation-and-case-numbers [Accessed 10 Dec 2020].

3 Policy Responses to COVID19. International monetary fund, 2020. Available: https://www.imf.org/en/Topics/imf-and-covid19/PolicyResponses-to-COVID-19 [Accessed 15 Dec 2020].

4 Di Renzo L, Gualtieri P, Pivari F, et al. Eating habits and lifestyle changes during COVID-19 lockdown: an Italian survey. $J$ Trans/ Med 2020;18:229.

5 Moore SA, Faulkner G, Rhodes RE, et al. Impact of the COVID-19 virus outbreak on movement and play behaviours of Canadian children and youth: a national survey. Int J Behav Nutr Phys Act 2020;17:85

6 Chopra S, Ranjan P, Singh V, et al. Impact of COVID-19 on lifestylerelated behaviours- a cross-sectional audit of responses from nine hundred and ninety-five participants from India. Diabetes Metab Syndr 2020;14:2021-30.

7 Di Renzo L, Gualtieri P, Cinelli G, et al. Psychological aspects and eating habits during COVID-19 home confinement: results of EHLCCOVID-19 Italian online survey. Nutrients 2020;12:2152.

8 Deschasaux-Tanguy M, Druesne-Pecollo N, Esseddik Y. Diet and physical activity during the COVID-19 lockdown period (March-May 2020): results from the French NutriNet-Sante cohort study. medRxiv 2020.

9 Carroll N, Sadowski A, Laila A, et al. The impact of COVID-19 on health behavior, stress, financial and food security among middle to high income Canadian families with young children. Nutrients 2020;12:2352.

10 Dunton GF, Do B, Wang SD. Early effects of the COVID-19 pandemic on physical activity and sedentary behavior in children living in the U.S. BMC Public Health 2020;20:1351.

11 Xiong J, Lipsitz O, Nasri F, et al. Impact of COVID-19 pandemic on mental health in the general population: a systematic review. $J$ Affect Disord 2020;277:55-64.

12 Serafini G, Parmigiani B, Amerio A, et al. The psychological impact of COVID-19 on the mental health in the general population. QJM 2020;113:531-7.

13 Luo M, Guo L, Yu M, et al. The psychological and mental impact of coronavirus disease 2019 (COVID-19) on medical staff and general public - A systematic review and meta-analysis. Psychiatry Res 2020;291:113190.

14 Rajkumar RP. COVID-19 and mental health: a review of the existing literature. Asian J Psychiatr 2020;52:102066.

15 Dubey S, Biswas P, Ghosh R, et al. Psychosocial impact of COVID-19. Diabetes Metab Syndr 2020;14:779-88.

16 Kirby T. Evidence mounts on the disproportionate effect of COVID-19 on ethnic minorities. Lancet Respir Med 2020;8:547-8.

17 White C, Nafilyan V. Coronavirus (COVID-19) related deaths by ethnic group, England and Wales: 2 March 2020 to 10 April 2020. office for national statistics, 2020. Available: https://www.ons.gov.uk/peoplepo pulationandcommunity/birthsdeathsandmarriages/deaths/articles/ coronavirusrelateddeathsbyethnicgroupenglandandwales/2march20 20to10april2020 [Accessed 28 Dec 2020].

18 World Health Organization. Health of migrants: resetting the agenda. Report of the 2nd global consultation, Colombo, Sri Lanka, 21-23 Feb 2017. Geneva: international organization for migration, 2017. Available: https://publications.iom.int/system/files/pdf/gc2_srilanka report_2017.pdf [Accessed 28 Dec 2020].

19 Goeman D, King J, Koch S. Development of a model of dementia support and pathway for culturally and linguistically diverse communities using co-creation and participatory action research. BMJ Open 2016;6:e013064.

20 Sze S, Pan D, Nevill CR, et al. Ethnicity and clinical outcomes in COVID-19: a systematic review and meta-analysis. EClinicalMedicine 2020;29:100630.

21 Wild A, Kunstler B, Goodwin D. Communicating COVID-19 health information to culturally and linguistically diverse (CALD) communities: the importance of partnership, co-design, and behavioural and implementation science. MetaArXiv 2020.

22 Australia's health 2018. Culturally and linguistically diverse populations. Australian Institute of health and welfare. Australian Government, 2018. Available: https://www.aihw.gov.au/getmedia/ f3ba8e92-afb3-46d6-b64c-ebfc9c1f945d/aihw-aus-221-chapter-5-3. pdf.aspx [Accessed 20 Dec 2020].
23 McCaffery KJ, Dodd RH, Cvejic E, et al. Health literacy and disparities in COVID-19-related knowledge, attitudes, beliefs and behaviours in Australia. Public Health Res Pract 2020;30:e30342012.

24 Ali SH, Foreman J, Tozan Y, et al. Trends and predictors of COVID-19 information sources and their relationship with knowledge and beliefs related to the pandemic: nationwide cross-sectional study. JMIR Public Health Surveill 2020;6:e21071.

25 Wang P-W, Lu W-H, Ko N-Y, et al. COVID-19-Related information sources and the relationship with confidence in people coping with COVID-19: Facebook survey study in Taiwan. J Med Internet Res 2020;22:e20021.

26 Wen LM, Rissel C, Baur LA, et al. A 3-Arm randomised controlled trial of communicating healthy beginnings advice by telephone (ChAT) to mothers with infants to prevent childhood obesity. BMC Public Health 2017;17:79.

27 Ekambareshwar M, Mihrshahi S, Wen LM, et al. Facilitators and challenges in recruiting pregnant women to an infant obesity prevention programme delivered via telephone calls or text messages. Trials 2018;19:494.

28 Wen LM, Rissel C, Xu H, et al. Effects of Telephone and Short Message Service Support on Infant Feeding Practices, "Tummy Time," and Screen Time at 6 and 12 Months of Child Age: A 3-Group Randomized Clinical Trial. JAMA Pediatr 2020;174:657-64.

29 Kroenke K, Spitzer RL, Williams JBW, et al. An ultra-brief screening scale for anxiety and depression: the PHQ-4. Psychosomatics 2009:50:613-21.

30 Centre for Epidemiology and Research. 2006 report on adult health from the new South Wales population health survey. Sydney: NSW department of health, Australia, 2007. Available: https://www.health. nsw.gov.au/surveys/adult/Publications/adults-06.pdf [Accessed 15 Dec 2020].

31 Rossell SL, Neill E, Phillipou A, et al. An overview of current mental health in the general population of Australia during the COVID-19 pandemic: results from the COLLATE project. Psychiatry Res 2021;296:113660.

32 Lupton D. Timeline of COVID-19 in Australia: the first year. Available: https://deborahalupton.medium.com/timeline-of-covid-19-inaustralia-1f7df6ca5f23 [Accessed 20 Jan 2021].

33 Thibaut F, van Wijngaarden-Cremers PJM. Women's Mental Health in the Time of Covid-19 Pandemic. Front Glob Womens Health 2020;1:Article588372.

34 Henrich N, Holmes B. Communicating during a pandemic: information the public wants about the disease and new vaccines and drugs. Health Promot Pract 2011;12:610-9.

35 Perrin A, Anderson M. Share of U.S. adults using social media, including Facebook, is mostly unchanged since 2018. Pew research center, 2020. Available: https://www.pewresearch.org/fact-tank/ 2019/04/10/share-of-u-s-adults-using-social-media-includingfacebook-is-mostly-unchanged-since-2018/ [Accessed 25 Jan 2021].

36 Park S, Fisher C, Lee JY. COVID-19: Australian news and misinformation. University of Canberra. Available: https://apo.org au/sites/default/files/resource-files/2020-07/apo-nid306728.pdf [Accessed 18 Jan 2021].

37 Spencer SH. False claims of nationwide lockdown for COVID-19. Factcheck, 2020. Available: https://www.factcheck.org/2020/03/ false-claims-of-nationwide-lockdown-for-covid-19/ [Accessed 20 Dec 2020].

38 Mental Health Commission. Understanding experiences and impacts of COVID-19 on individuals with mental health and AOD issues from CaLD communities, 2020. Available: https://www.mhc.wa. gov.au/media/3413/201016-mhc20-80370-impact-of-covid-19-onindividuals-cald-communities-final-attachment.pdf [Accessed $20 \mathrm{Dec}$ 2020].

39 Sakya SM, Scoy LJV, Garman JC, et al. The impact of COVID-19related changes in media consumption on public knowledge: results of a cross-sectional survey of Pennsylvania adults. Curr Med Res Opin 2021;37:911-5.

40 Food and nutrition tips during self-quarantine. WHO, 2020. Available: http://www.euro.who.int/en/health-topics/health-emergencies/ coronavirus-covid-19/novel-coronavirus-2019-ncov-technicalguidance/food-and-nutrition-tips-during-self-quarantine [Accessed 16 Jan 2021].

41 Liu J, Davidson E, Bhopal R, et al. Adapting health promotion interventions to meet the needs of ethnic minority groups: mixedmethods evidence synthesis. Health Technol Assess 2012;16:1-469. 